\title{
Conformation sensitive charge transport in conjugated polymers
}

Mattias Andersson, Svante Hedstrom and Petter Persson

\author{
Linköping University Post Print
}

\section{Tweet}

N.B.: When citing this work, cite the original article.

Original Publication:

Mattias Andersson, Svante Hedstrom and Petter Persson, Conformation sensitive charge transport in conjugated polymers, 2013, Applied Physics Letters, (103), 21, 213303.

http://dx.doi.org/10.1063/1.4832075

Copyright: American Institute of Physics (AIP)

http://www.aip.org/

Postprint available at: Linköping University Electronic Press

http://urn.kb.se/resolve?urn=urn:nbn:se:liu:diva-102780 


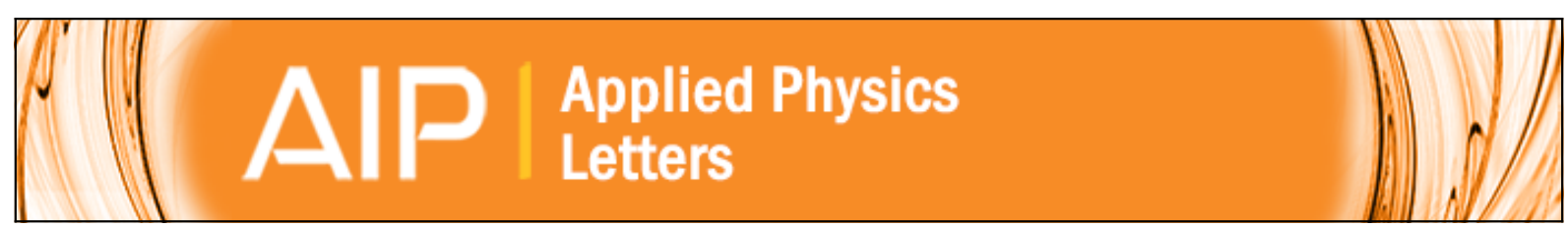

\section{Conformation sensitive charge transport in conjugated polymers}

L. Mattias Andersson, Svante Hedström, and Petter Persson

Citation: Applied Physics Letters 103, 213303 (2013); doi: 10.1063/1.4832075

View online: http://dx.doi.org/10.1063/1.4832075

View Table of Contents: http://scitation.aip.org/content/aip/journal/apl/103/21?ver=pdfcov

Published by the AIP Publishing

$\underset{\text { Aoublonise }}{A}$ Re-register for Table of Content Alerts

Create a profile.

Sign up today! 


\title{
Conformation sensitive charge transport in conjugated polymers
}

\author{
L. Mattias Andersson, ${ }^{1, a)}$ Svante Hedström, ${ }^{2}$ and Petter Persson ${ }^{2}$ \\ ${ }^{1}$ Department of Physics, Chemistry and Biology (IFM), Linköping University, Linköping SE-581 83, Sweden \\ ${ }^{2}$ Division of Theoretical Chemistry, Lund University, P.O. Box 124, SE-221 00 Lund, Sweden
}

(Received 16 October 2013; accepted 3 November 2013; published online 21 November 2013)

Temperature dependent charge carrier mobility measurements using field effect transistors and density functional theory calculations are combined to show how the conformation dependent frontier orbital delocalization influences the hole- and electron mobilities in a donor-acceptor based polymer. A conformationally sensitive lowest unoccupied molecular orbital results in an electron mobility that decreases with increasing temperature above room temperature, while a conformationally stable highest occupied molecular orbital is consistent with a conventional hole mobility behavior and also proposed to be one of the reasons for why the material works well as a hole transporter in amorphous bulk heterojunction solar cells. (C) 2013 AIP Publishing LLC. [http://dx.doi.org/10.1063/1.4832075]

Organic semiconductors are rapidly finding their way into more and more applications, and new materials are developed at an ever-increasing rate. One such material, the donor-acceptor polymer poly[2,3-bis-(3-octyloxyphenyl) quinoxaline-5,8-diyl-alt-thiophene-2,5-diyl] (TQ1), has been developed for use in organic bulk heterojunction solar cells where it performs admirably with respect to its physical limits. ${ }^{1}$ There are hard (material specific) limits on achievable solar cell efficiencies in the form of, e.g., optical band gap and relative donor-acceptor energy level positions. There are also much more commonly encountered soft performance limits, usually related to morphology and phase structure in the form of, e.g., too large phase separation or isolated clusters of one or both of the phases. Only a very small fraction of the multitude of developed materials has come close to the hard efficiency limits. ${ }^{1,2}$ Interestingly, fundamentally different morphologies and phase structures have proven to be able to achieve this; poly(3-hexylthiophene) (P3HT) for instance, relies on crystallization, ${ }^{3}$ while TQ1 is largely amorphous. ${ }^{4}$ Figuring out why certain materials work well, and why others do not, is one of the most rewarding paths to progress. P3HT has been thoroughly investigated and covers the general behavior of crystalline materials well, but there has been considerably less work done on amorphous materials.

To a very large extent, the achieved performance of a specific material system simply reflects the amount of effort spent on synthesis refinement and device optimization. There are, however, certainly differences in how easy the optimization process is for a particular material, and some materials are simply too difficult to be worthwhile. From an application point of view, it is reasonable to believe that easy to optimize materials are more robust and suitable for commercial applications so there are plenty of reasons to consider not only the maximum achievable performance of a material but also how easy it is to get there. Here, temperature dependent charge carrier mobility measurements together with density functional theory (DFT) calculations give insight into some of the fundamental advantages of TQ1. Essentially, this is related to the ability of TQ1 to perform

${ }^{a)}$ E-mail: matan@ifm.liu.se well in an amorphous state, which in turn is linked to the properties of the frontier molecular orbitals and their (in-) sensitivity to conformational changes. These findings can aid in the screening process of new materials and also indicate a possibility for easily fabricated devices with unconventional electronic functions.

Charge transport in conjugated materials occurs in the frontier orbitals, where charges can be delocalized. The degree of delocalization varies, however, and is rarely, if ever, sufficient for ballistic transport. When localization of a charge carrier occurs, activation is necessary for it to continue on its path. Localization can be due to the chemical nature of the molecule, as well as to, e.g., structural, conformational, or packing defects. The soft nature of these materials frequently also causes self-localization of charges due to reorganization. Almost all conjugated materials are dominated by such an activated charge carrier transport. One very important consequence of activated charge transport that is relevant for the present discussion is that the charge carrier mobility in such a system increases with increasing temperature in an Arrhenius-like fashion.

The intimate relationship between the frontier orbital properties and charge transport does, however, make the mobility potentially sensitive to conformational changes and phase transitions since these are in turn known to affect the orbitals. ${ }^{4,5}$ Information about the electronic structure of a material can thus aid in the interpretation of experimental charge carrier transport data. One of the most well established ways to calculate the electronic structure is with DFT. ${ }^{6}$ DFT is capable of predicting electronic, optical, and structural properties. Due to computational limitations, longer polymer chains are not possible to study, but qualitative explanatory information can be obtained from oligomer calculations with the help of extrapolation techniques that exist for quantitative polymeric predictions. TQ1 has previously been studied with DFT, including frontier orbital analysis, optical absorption profile, and geometrical structure. ${ }^{7}$

As cast TQ1 films have a relatively low field effect transistor (FET) hole mobility, on the order of $10^{-5} \mathrm{~cm}^{2} \mathrm{~V}^{-1} \mathrm{~s}^{-1}$. In contrast to many other donor-acceptor polymers, it also exhibits an electron mobility, albeit about one order of 
magnitude lower than the hole mobility. Annealing above the glass transition temperature has no significant influence on the hole mobility but drastically increases the room temperature electron mobility by almost two orders of magnitude. Figure 1(a) shows the hole and electron mobility as a function of increasing temperature for an as cast film as well as for the same film after annealing above the glass transition temperature. The temperature dependence of the hole mobility is as expected, i.e., the mobility increases with increasing temperature up to the glass transition temperature. Interestingly enough, the electron mobility in the annealed sample does not follow the same trend even though both mobilities are measured simultaneously and on the same device. Further in-situ measurements during consecutive temperature scans after sufficient annealing yield identical results within the experimental accuracy. In all cases, it is possible to identify the glass transition temperature as a change in the temperature dependence. Extending the observed temperature range reveals that the electron mobility does behave conventionally at lower temperatures and that both mobilities have similar activation
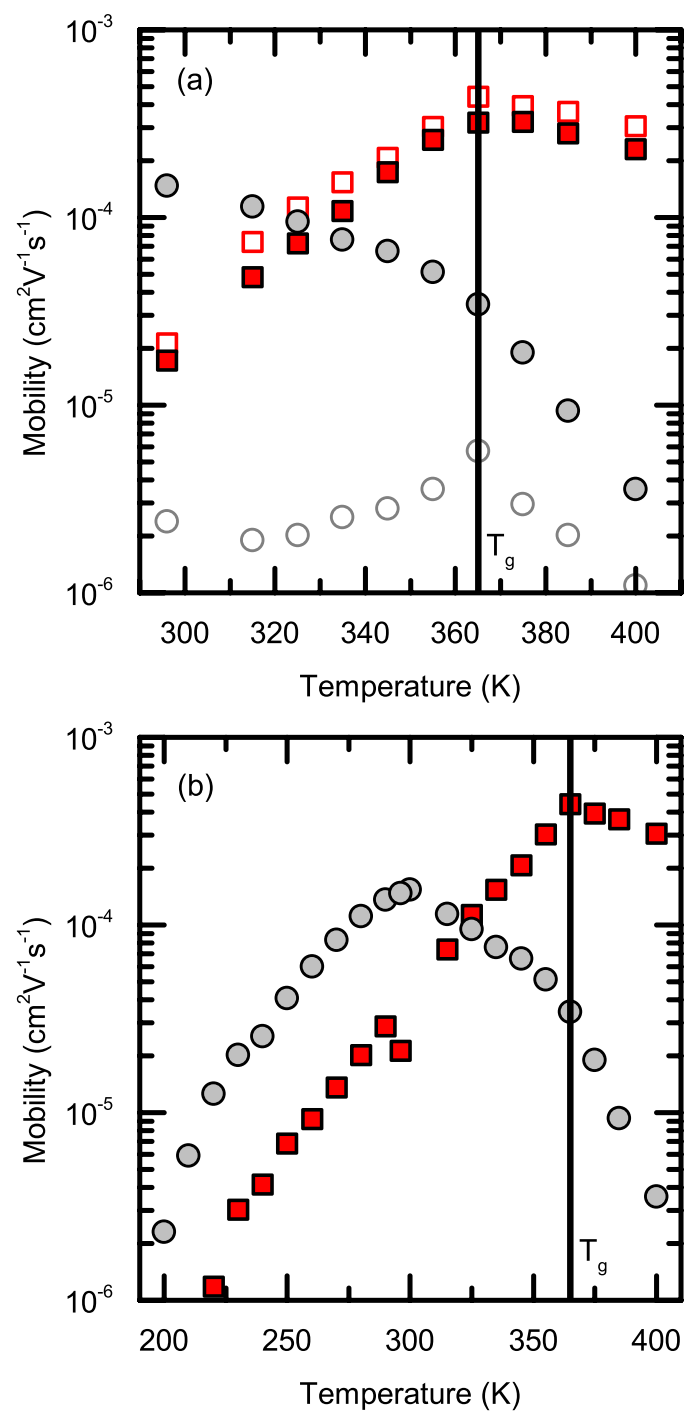

FIG. 1. Hole (squares) and electron (circles) mobility versus temperature for an as cast (open symbols) TQ1 film and the same film after annealing (solid symbols) (a), and mobility versus temperature for an extended temperature range after annealing (b). A vertical line indicates the glass transition temperature in both (a) and (b). energies, i.e., similar slopes in the Arrhenius representation. This can be seen in, and inferred from, Figure 1(b).

Experimental evidence from, e.g., molecular weight dependent charge transport measurements suggests that the charge transport in this type of amorphous polymer occur predominately along the polymer chains. ${ }^{4}$ Extended delocalization along the chain of the frontier molecular orbitals is thus very important for efficient charge transport. Quantum chemical calculations on other donor-acceptor polymers have shown that the highest occupied molecular orbital (HOMO), where hole transport occurs, is well delocalized along the whole chain, while the lowest unoccupied molecular orbital (LUMO) is localized to one of the repeat units. ${ }^{8}$ Accordingly, those materials have a decent hole mobility in FETs, but show no evidence of electron transport. ${ }^{9}$

DFT calculations on optimized TQ1 oligomers reveal that both frontier orbitals are delocalized, which is consistent with the ambipolar properties of the material. While high hole mobilities are relatively common in donor-acceptor polymers, high electron mobilities are more unusual. This could possibly be explained by the fact that the LUMO, as is the case for TQ1, tends to be associated with the acceptor unit. In contrast to many other donor-acceptor polymers, the donor unit of TQ1 is small; a single thiophene unit, whereas most other materials have considerably larger donor units. The small size of the thiophene unit does not spatially isolate the acceptor units and allows for an unbroken LUMO across the backbone under favorable circumstances, but only barely; the delocalization of the LUMO is strongly conformation dependent. Figure 2 shows calculated HOMO and LUMO orbitals for partially optimized TQ1 dimers with representative, fixed, dihedral angles between two consecutive quinoxaline units. The TQ1 LUMO displays good delocalization for the more planar structure yielded by the minimum energy interquinoxaline dihedral angle of $53^{\circ}$, as well as those of $133^{\circ}$ and $183^{\circ}$, whereas the more twisted dihedral angles of, e.g., $3^{\circ}, 23^{\circ}$, and $93^{\circ}$ effectively break the LUMO delocalization. Although calculations on full films or even complete polymer chains are not possible, the energy minimized structure in vacuum does have a delocalized LUMO and it is reasonable to assume that such a conformation will be more favored in the annealed films compared to the as cast films.

The different temperature dependencies of the hole and electron mobilities seen above room temperature in Figure 1 are thus consistent with the properties of the frontier orbitals. While the HOMO is always delocalized, the delocalization of the LUMO is strongly dependent on the dihedral angle. A higher thermal energy permits the polymer to adopt less energetically favorable conformations, with more twisted and kinked backbones, indicating a predisposition for macroscopic non-uniformity, and a high sensitivity of the electrical properties to conformational changes. Physically, the proposed conformational changes in TQ1 films at increased temperatures is consistent with the fact that they swell by about $4 \%$ (determined by ellipsometry) between room temperature and the glass transition temperature. Such a swelling is bound to be associated with some conformational variations, and due to the very strong correlation between conformation and LUMO delocalization it is reasonable to 

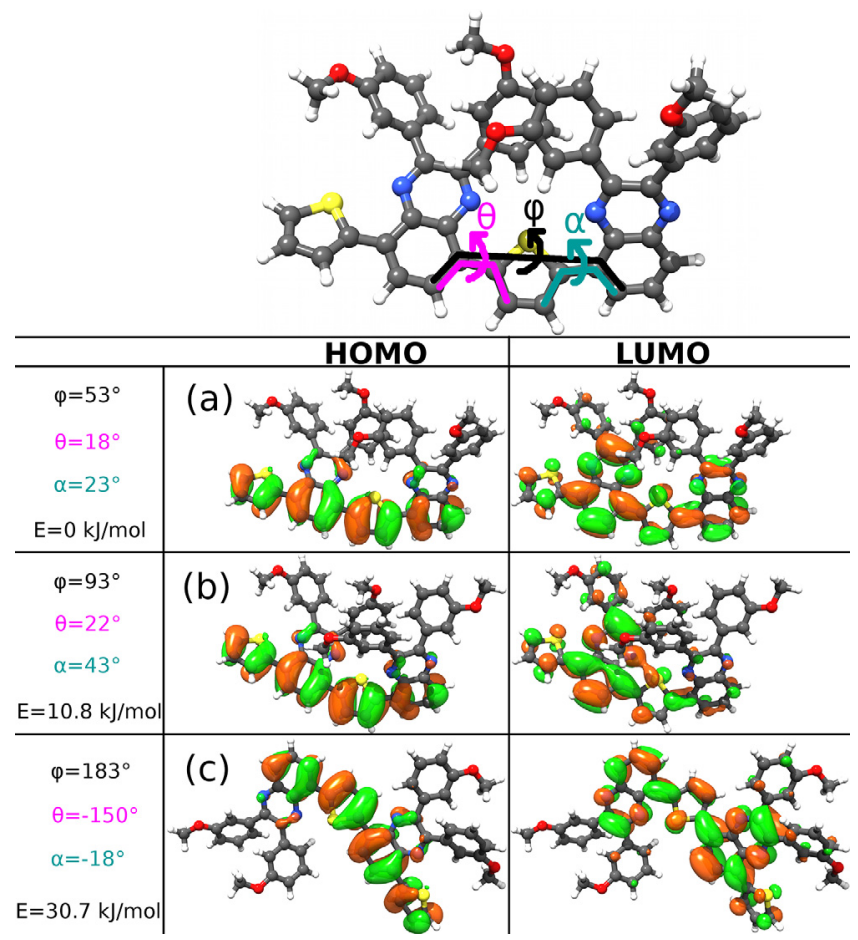

FIG. 2. TQ1 frontier orbitals for the fully optimized TQ1 dimer (a), as well as for two representative cases with imposed interquinoxaline dihedral angles, $\varphi$, ((b) and (c)), exhibiting decent, poor, and good LUMO delocalization, respectively. Also shown are the resulting thiophene-quinoxaline dihedral angles $\theta$ and $\alpha$. The total system energy is given relative to the minimum energy conformation. Calculated at the $\omega \mathrm{B} 97 \mathrm{XD} / 6-311+\mathrm{G}(2 \mathrm{~d}, \mathrm{p}) / / 6-$ $31 \mathrm{G}(\mathrm{d}, \mathrm{p})$ level of theory.

conclude that the electron mobility will be (negatively) influenced. It should be noted that it might not be localization per se that is the sole cause the observed behavior; spatial LUMO variations will give rise to an increased energetic disorder, which also affects the mobility negatively.

Based on these results, it can be speculated that part of the reason for the successful application of TQ1 in amorphous bulk heterojunction solar cells may be due to the conformational stability of the HOMO delocalization. Because of this stability, even the rather unimpressive hole mobility of TQ1 is capable of high quantum efficiencies and fill factors since the charge transport is relatively homogenous throughout the film and without isolated low-mobility regions. Had the properties of the HOMO and the LUMO been exchanged however, amorphous structures would probably no longer be viable. Frontier orbital delocalization considerations have already been empirically incorporated in some of the best performing polymers, where abundant use of fused rings in their backbones ensures a favorable electronic structure with extensive frontier orbital delocalization. ${ }^{10}$ While obviously a possible strategy, the synthesis of such systems is frequently more complex and costly than that of simpler structures. These results show that a forced conformation is not strictly necessary for good charge transport, and that it is possible to find simpler and cheaper materials with the desired frontier orbital properties.

Outside of solar cell applications, there might be new and interesting applications for materials that shift between being dominated by hole or electron mobility at different temperatures. Figure 1(a) shows that in going from room temperature to just below the glass transition temperature in annealed TQ1 films, there is an almost symmetric switch between the magnitudes of the hole- and electron mobilities. One of the drawbacks of organic materials is their stability. By using differential signals from a single material it might, for instance, be possible to mitigate such shortcomings. With rational material design, either frontier orbital's properties should also be possible to tailor for specific needs.

To conclude, differences in the temperature dependence of the hole- and electron mobilities in TQ1 have been linked to conformational dependencies of the frontier orbital properties through DFT calculations. The degree of HOMO delocalization in TQ1 is independent of its conformation, which gives it a stable hole mobility and allows it to function well in amorphous bulk heterojunction solar cells. On the other hand, the LUMO delocalization depends strongly on the conformation, which results in an unconventional electron mobility temperature dependence. Instead of an Arrhenius like increase in electron mobility with increasing temperature it starts to decrease well below the glass transition temperature. This behavior is explained by the conformationally sensitive LUMO delocalization revealed by DFT calculations since higher temperatures induce a higher degree of twisting of the polymer chains. Strongly conformation dependent frontier orbital delocalization is therefore proposed to be detrimental to the efficiency of amorphous bulk heterojunction solar cells, while at the same time being of interest for new applications where temperature dependent variations in the polarity of the dominant carrier type can be exploited.

The authors would like to thank E. Wang and M. R. Andersson for the supply of TQ1. L.M.A. wishes to thank C. Müller and J. Bergqvist for fruitful discussions. NSC and LUNARC are acknowledged for computer resources used. This work was funded by the Swedish Energy Agency and the Swedish Research Council.

${ }^{1}$ E. G. Wang, L. T. Hou, Z. Q. Wang, S. Hellstrom, F. L. Zhang, O. Inganas, and M. R. Andersson, Adv. Mater. 22(46), 5240 (2010).

${ }^{2}$ P. Schilinsky, C. Waldauf, and C. J. Brabec, Appl. Phys. Lett. 81(20), 3885 (2002).

${ }^{3}$ C. Muller, T. A. M. Ferenczi, M. Campoy-Quiles, J. M. Frost, D. D. C. Bradley, P. Smith, N. Stingelin-Stutzmann, and J. Nelson, Adv. Mater. 20(18), 3510 (2008).

${ }^{4}$ L. M. Andersson, Org. Electron. 12(2), 300 (2011).

${ }^{5}$ C. Muller, J. Bergqvist, K. Vandewal, K. Tvingstedt, A. S. Anselmo, R. Magnusson, M. I. Alonso, E. Moons, H. Arwin, M. Campoy-Quiles, and O. Inganas, J. Mater. Chem. 21(29), 10676 (2011).

${ }^{6}$ B. Grimm, C. Risko, J. D. Azoulay, J. L. Bredas, and G. C. Bazan, Chem. Sci. 4(4), 1807 (2013); L. Yang, J. K. Feng, A. M. Ren, and J. Z. Sun, Polymer 47(4), 1397 (2006); S. S. Zade, N. Zamoshchik, and M. Bendikov, Acc. Chem. Res. 44(1), 14 (2011).

${ }^{7}$ S. Hedstrom and P. Persson, J. Phys. Chem. C 116(51), 26700 (2012); R. Kroon, A. Lundin, C. Lindqvist, P. Henriksson, T. T. Steckler, and M. R. Andersson, Polymer 54(4), 1285 (2013); E. G. Wang, J. Bergqvist, K. Vandewal, Z. F. Ma, L. T. Hou, A. Lundin, S. Himmelberger, A. Salleo, C. Muller, O. Inganas, F. L. Zhang, and M. R. Andersson, Adv. Energy Mater. 3(6), 806 (2013).

${ }^{8}$ K. G. Jespersen, W. J. D. Beenken, Y. Zaushitsyn, A. Yartsev, M. Andersson, T. Pullerits, and V. Sundstrom, J. Chem. Phys. 121(24), 12613 (2004).

${ }^{9}$ L. M. Andersson, F. L. Zhang, and O. Inganas, Appl. Phys. Lett. 91(7), 071108 (2007).

${ }^{10}$ W. W. Li, K. H. Hendriks, W. S. C. Roelofs, Y. Kim, M. M. Wienk, and R. A. J. Janssen, Adv. Mater. 25(23), 3182 (2013). 